\title{
Design Rules for Charge-Transport Efficient Host Materials for Phosphorescent Organic Light-Emitting Diodes
}

Falk May, ${ }^{\dagger, \ddagger}$ Mustapha Al-Helwi, ${ }^{\ddagger},{ }^{\prime} \perp$ Björn Baumeier, ${ }^{\dagger}$ Wolfgang Kowalsky, ${ }^{\perp, \|}$ Evelyn Fuchs, ${ }^{\S}$ Christian Lennartz, ${ }^{* \neq}, \S$ and Denis Andrienko*, ${ }^{\dagger}$

${ }^{\dagger}$ Max Planck Institute for Polymer Research, Ackermannweg 10, 55128 Mainz, Germany
${ }^{\ddagger}$ Innovation Lab Heidelberg, Speyerer Strasse 4, 69115 Heidelberg, Germany
${ }^{\S}$ BASF SE, B009, 67056 Ludwigshafen, Germany
${ }^{\perp}$ Kirchhoff-Institut für Physik der Universität Heidelberg, Im Neuenheimer Feld 227, 69120 Heidelberg, Germany
${ }^{\|}$Institut für Hochfrequenztechnik, Technische Universität Braunschweig, Schleinitzstrasse 22, 38106 Braunschweig, Germany

Supporting Information

ABSTRACT: The use of blue phosphorescent emitters in organic light-emitting diodes (OLEDs) imposes demanding requirements on a host material. Among these are large triplet energies, the alignment of levels with respect to the emitter, the ability to form and sustain amorphous order, material processability, and an adequate charge carrier mobility. A possible design strategy is to choose a $\pi$-conjugated core with a

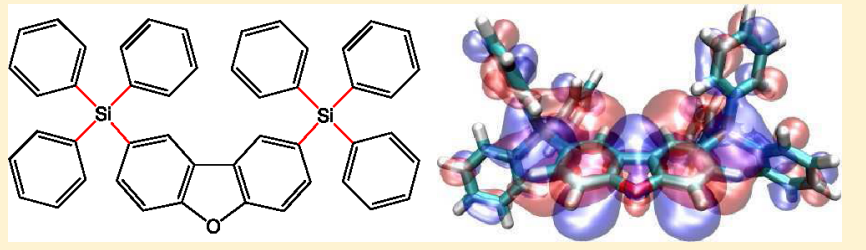
high triplet level and to fulfill the other requirements by using suitable substituents. Bulky substituents, however, induce large spatial separations between conjugated cores, can substantially reduce intermolecular electronic couplings, and decrease the charge mobility of the host. In this work we analyze charge transport in amorphous 2,8-bis(triphenylsilyl)dibenzofuran, an electron-transporting material synthesized to serve as a host in deep-blue OLEDs. We show that mesomeric effects delocalize the frontier orbitals over the substituents recovering strong electronic couplings and lowering reorganization energies, especially for electrons, while keeping energetic disorder small. Admittance spectroscopy measurements reveal that the material has indeed a high electron mobility and a small Poole-Frenkel slope, supporting our conclusions. By linking electronic structure, molecular packing, and mobility, we provide a pathway to the rational design of hosts with high charge mobilities.

\section{INTRODUCTION}

Organic light-emitting diodes (OLEDs) have recently entered the market of flat panel displays and lighting applications. ${ }^{1,2}$ In spite of this successful commercialization, the field still has a number of open issues, such as insufficient stability ${ }^{3}$ of OLEDs based on deep-blue $(\lambda<460 \mathrm{~nm})$ emitters. ${ }^{4,5}$

In a prototypical phosphorescent OLED, holes and electrons are injected from electrodes on opposite sides into transport and blocking layers that provide a balanced charge transport into an emission layer (EML). The EML itself consists of a charge-transporting organic semiconductor and an organometallic emitter that allows for triplet harvesting. To avoid triplet quenching and triplet-triplet annihilation, the chargetransporting material as the majority component (host) is doped with the emitter. An exciton can be formed on a host molecule with a subsequent energy transfer to the dopant. Alternatively, one of the charge carriers can be trapped on the emitter and form a neutral exciton on-site by attracting a charge of the opposite sign. This direct charge transfer to the emitter is argued to lead to more efficient OLEDs than excitation by energy transfer. $^{6-9}$ The balance between the two routes is determined by the respective energy level alignment of the emitter and the host, and can therefore be rationally designed. However, due to the large band gap of the deep-blue emitter and the resulting high triplet energy, the number of promising compatible host materials is limited, since an even higher triplet energy is necessary for the host to ensure trapping of the exciton on the emitter. ${ }^{10,11}$ Among possible charge-transporting units fulfilling this criterion are dibenzofurans and $N$-phenylcarbazoles. ${ }^{12}$ Substituents must be attached to these materials to prevent crystallization, ${ }^{13}$ suppress emitter aggregation, and optimize their molecular weight for vacuum deposition. The main role of the substituents, however, is to adjust the relative positions of electron- and hole-transporting levels as well as singlet and triplet excited states of the host to those of the emitter. The relative level alignment, together with the processes occurring in the EML, is shown in Figure 1a,b for a hole-conducting emitter. Synthetically, level adjustment can be achieved by inductive effects ${ }^{14}$ when strongly electronegative substituents (e.g., trifluoromethyl) are used. An alternative approach is to exploit the mesomeric effect ${ }^{14}$ when the frontier orbital densities delocalize (by using, e.g., triphenylsilyl substituents).

Apart from a suitable level alignment, processability, amorphousness, and stability, an adequate charge carrier

Received: June 6, 2012

Published: July 30, 2012 
(a)
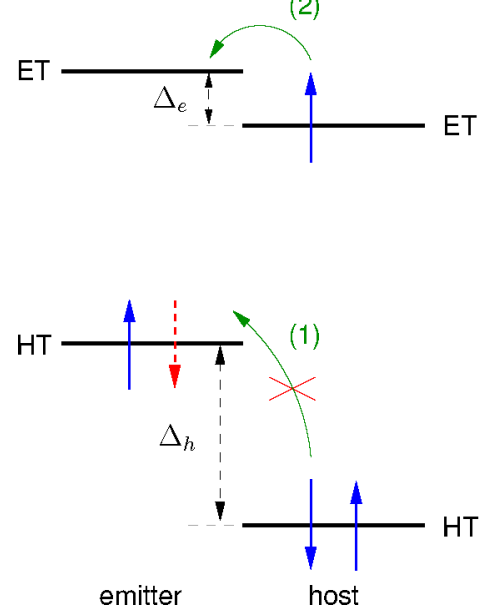

(b)

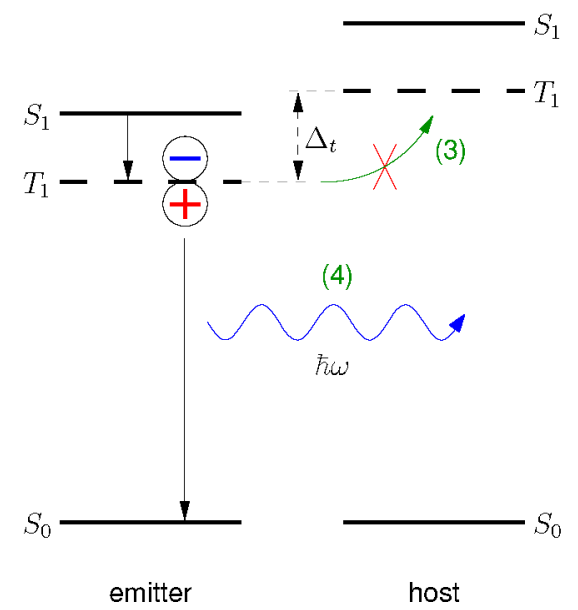

(c)

(d)
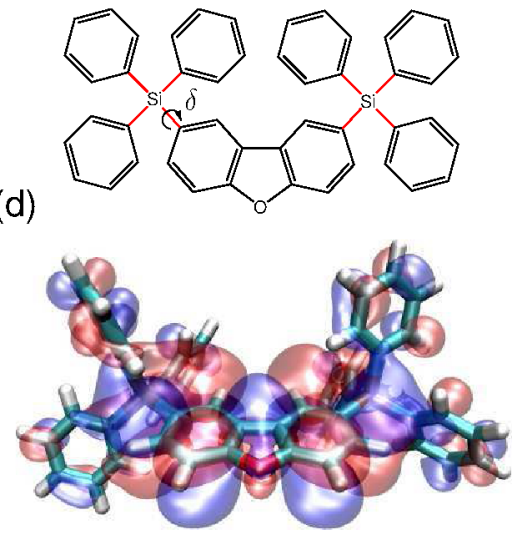

Figure 1. (a) Hole- (HT) and electron-transport (ET) levels of emitter and host in the EML. (b) Energy levels of singlets $(S)$ and triplets $(T)$ of the emitter emission spectrum and the host excitation spectrum. The following processes are shown assuming a hole (electron)-transporting emitter (host): (1) Hole transfer from emitter to host is avoided by a large barrier, $\Delta_{\mathrm{h}}$. (2) Electron transfer is prevented by the barrier $\Delta_{\mathrm{e}}$. In order to ensure exciton formation on the emitter, $\Delta_{\mathrm{h}}$ has to be larger than $\Delta_{\mathrm{e}}$ and the Coulomb attraction between the hole on the emitter and the electron on the neighboring host should be capable of overcoming $\Delta_{\mathrm{e}}$, attracting the electron to the emitter cation. In practice $\Delta_{\mathrm{h}} \approx 1 \mathrm{eV}$ and $\Delta_{\mathrm{e}} \approx 0.3 \mathrm{eV}$. (3) Backtransfer of the exciton from the emitter to the host is avoided by the barrier $\Delta_{\mathrm{t}}$ for triplet excitons. This also prevents re-absorption of an emitted photon by the host. (4) Carrier recombination and emission of blue light. (c) Chemical structure of BTDF with eight "soft" dihedrals (red). (d) Isosurfaces of the LUMO. An isovalue of \pm 0.007 au allows to visualize small fractions of orbital density on the substituents.

(a)

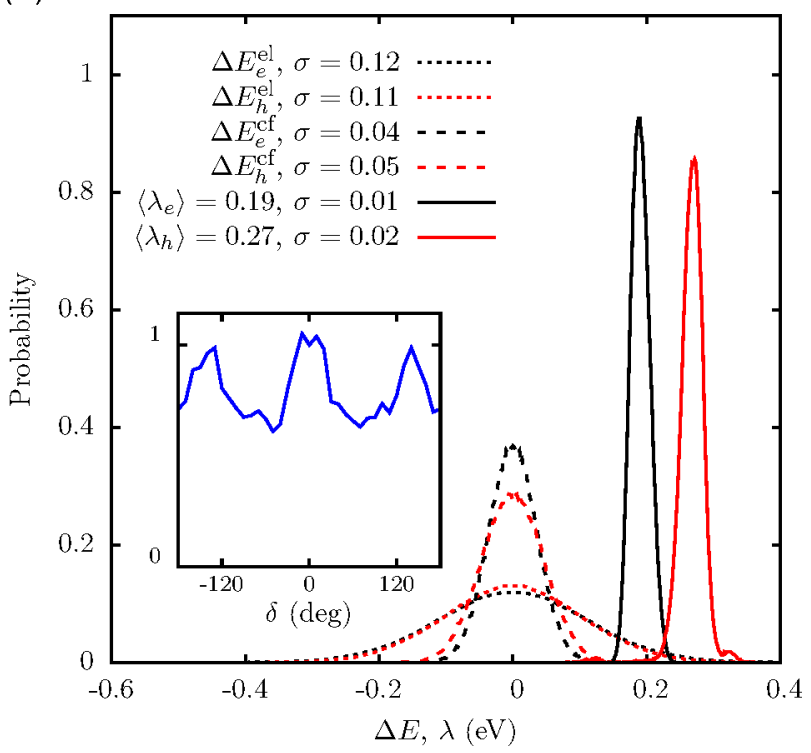

(b)

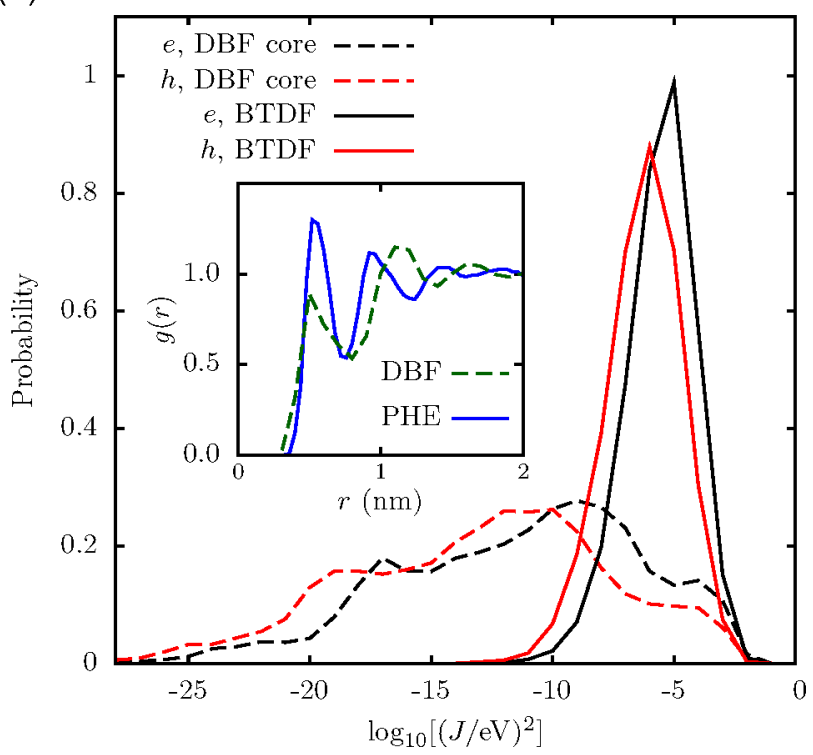

Figure 2. (a) Distributions of differences in electrostatic energies including polarization, $\Delta E_{\mathrm{e}}^{\mathrm{el}} \mathrm{h}$ (from a neighbor list), conformational energies, $\Delta E_{\mathrm{e}(\mathrm{h})}^{\mathrm{cf}}$, and reorganization energies, $\lambda_{\mathrm{e}(\mathrm{h})}$, for electrons (holes). Mean and variance $\sigma$ given in $\mathrm{eV}$. Inset shows the distribution of the dihedral angle $\delta$ introduced in Figure 1c. (b) Distributions of the logarithm of the transfer integral $J$ for electron and hole transport constructed from diabatic states based on dibenzofuran (DBF) core or using the whole molecule (BTDF). The same neighbor list and morphology of 4096 BTDF molecules was used for all distributions. Inset: Radial distribution functions $g(r)$ of the centers of mass of the DBF core (DBF) and of phenyl rings of the triphenylsilyl groups (PHE).

mobility of the host is required in order to prevent ohmic losses. Due to its complexity, the effect of the attached substituents on charge carrier mobility has rarely been addressed. It is, however, obvious that bulky substituents can lead to large spatial separations of $\pi$-conjugated systems of neighboring molecules. Since electronic couplings decrease exponentially with intermolecular separations, one might expect very poor charge carrier mobility of the host. The aim of this study is to show that the use of the mesomeric effect can remedy the situation by delocalizing the frontier orbitals over the substituents. To do this, we perform a combined experimental (admittance spectroscopy) and computer simulation study of charge transport in 2,8-bis(triphenylsilyl)dibenzofuran (BTDF), a typical electron-conducting host used in combination with hole-conducting deep-blue emitters. ${ }^{5}$ The maximal external quantum efficiencies of such OLEDs are above $17 \%$ (see the Supporting Information for the device charateristics). 
The chemical structure of BTDF is shown in Figure 1c. The two triphenylsilyl groups are attached to a dibenzofuran core, which lowers its electron-transport level below that of the emitter. The details of the synthesis are described in the Supporting Information.

\section{RESULTS}

2.1. Computer Simulations. To relate charge carrier mobility to the chemical structure, atomistic molecular dynamics (MD) is used to simulate material morphologies. Then the high-temperature limit of Marcus theory ${ }^{15,16}$ is employed to evaluate charge-transfer rates between molecules $i$ and $j$ according to

$$
\omega_{i j}=\frac{J_{i j}^{2}}{\hbar} \sqrt{\frac{\pi}{\lambda_{i j} k_{\mathrm{B}} T}} \exp \left[-\frac{\left(\Delta E_{i j}-\lambda_{i j}\right)^{2}}{4 \lambda_{i j} k_{\mathrm{B}} T}\right]
$$

where $T$ is the temperature, $J_{i j}$ is the electronic coupling element, or transfer integral, and $\Delta E_{i j}$ is the site energy difference which has contributions due to an applied electric field, electrostatics including polarization, $\Delta E^{\mathrm{el}}$, and internal energy differences due to molecular conformations, $\Delta E^{\mathrm{cf}}$. Finally, $\lambda_{i j}$ is the reorganization energy which is dominated by intramolecular contributions due to a small Pekar factor. ${ }^{17}$ More information about the transport parameters can be found in the Supporting Information.

The rates and molecular centers of mass are used in kinetic Monte Carlo simulations to solve the master equation for a charge drift-diffusing in a box with periodic boundary conditions in an applied electric field $F$. The charge carrier mobility is then determined as $\mu=\langle v\rangle / F$, where $\langle v\rangle$ is the averaged projection of the carrier velocity on the direction of the field.

Simulated mobilities are averaged over two MD snapshots, ten injection points, and six different spatial directions of the field. More details are given in the Supporting Information. Simulations are performed using the VOTCA package. ${ }^{17,18}$ This approach has been used to calculate mobility in columnar discotic mesophases, ${ }^{19-24}$ amorphous systems, ${ }^{25-28}$ selfassembled monoloayers, ${ }^{29}$ and conjugated polymers. ${ }^{30,31}$

An amorphous morphology of 4096 BTDF molecules is obtained by first annealing the system at $700 \mathrm{~K}$, well above the glass transition temperature, $T_{\mathrm{g}}=380 \mathrm{~K}$, followed by fast quenching to room temperature. The final length of the cubic box is $L=16 \mathrm{~nm}$. To determine intermolecular charge-hopping rates in this morphology, a neighbor list based on the closest approach of centers of mass between phenyl rings or dibenzofuran cores is constructed using a cutoff of $0.7 \mathrm{~nm}$. The parameters entering the rate expression eq 1 are then calculated for each molecular pair from the neighbor list.

Since BTDF has soft degrees of freedom, such as dihedrals $\delta$ in Figure 1c, molecules in the amorphous phase have different conformations. The distribution of this dihedral angle is shown in the inset of Figure 2a. These conformations are frozen on the time scale of charge transport (see the Supporting Information for details). Reorganization energies $\lambda_{i j}$ and internal energy differences $\Delta E_{i j}^{\mathrm{cf}}$ are therefore computed from potential energy surfaces of 512 molecules in neutral and charged states making use of density functional theory (DFT). We find a small variance in reorganization energies which does not affect the mobility. Hence, the mean values of $\lambda_{\mathrm{e}(\mathrm{h})}=0.19(0.27) \mathrm{eV}$ for electrons (holes) are used. Due to delocalization effects, ${ }^{32}$ attaching the triphenylsilyl groups decreases the reorganization energy (the dibenzofuran core has $\lambda_{\mathrm{e}(\mathrm{h})}^{\mathrm{DBF}}=0.27 \mathrm{eV}$ ) and hence increases electron-hopping rates.

The distributions of conformational energy differences, $\Delta E_{\mathrm{e}(\mathrm{h})}^{\mathrm{cf}}$, are of Gaussian type with a moderate variance of $\sigma_{\mathrm{e}}^{\mathrm{cf}}(\mathrm{h})$ $=0.04(0.05) \mathrm{eV}$ and are uncorrelated in space, which allows us to draw them from such a distribution in the larger box of 4096 molecules. Simulations are then additionally averaged over two realizations of this disorder.

Electrostatic contributions to site energy differences are calculated using partial charges for charged and neutral molecules in the ground state obtained from DFT. Polarization contributions are taken into account self-consistently using the Thole model ${ }^{33,34}$ with a cutoff of $3.5 \mathrm{~nm}$ between molecular centers of mass. This results in polarized electrostatic site energy differences, $\Delta E_{\mathrm{e}(\mathrm{h})}^{\mathrm{el}}$, that are Gaussian distributed with a variance of $\sigma_{\mathrm{e}(\mathrm{h})}^{\mathrm{el}}=0.12(0.11) \mathrm{eV}$ when computed from the neighbor list. Rather small energetic disorder and weak spatial correlations are due to small variations of atomic partial charges (local dipole moments) as well as the total dipole moment of BTDF of less than $1 \mathrm{D}$. Note that the attachment of the substituents does not affect the molecular dipole moment. All distributions are shown in Figure 2a.

The remaining ingredient entering the rate expression, eq 1 , is the transfer integral $J$, which relies on the definition of diabatic states of a pair of molecules. The latter are usually constructed from representative orbitals of the $\pi$-conjugated parts, since the effect of attached substituents on the diabatic states is rather small (e.g., in case of alkyl or glycol side chains $\left.{ }^{20,24,26}\right)$. Following this approach, the diabatic states are evaluated by substituting triphenylsilyl by a hydrogen (without modifying the rest of the morphology). Reorganization energies of the dibenzofuran core are used, and transfer integrals are then calculated on DFT level with the PBE functional and a TZVP basis set using the dimer projection method. ${ }^{35,36}$ The distribution of the logarithm of transfer integrals $J$ (see Figure $2 b$ ) for pairs of the neighbor list is very broad. This can be rationalized in terms of morphology, as the transfer integral depends exponentially on the intermolecular separation. The distance between the dibenzofuran cores is large due to the attached bulky substituents, as illustrated by the radial distribution function for centers of mass of dibenzofuran cores, shown in the inset of Figure $2 \mathrm{~b}$. The onset of this function is at ca. $0.5 \mathrm{~nm}$ and has a peak $g(r)>1$ at a separation larger than $1 \mathrm{~nm}$, which eventually leads to the broad distribution of $J$. The small number of high transfer integrals due to a few close-lying cores is apparently not sufficient for charge percolation. As a consequence, simulations predict low mobilities at experimentally relevant electric fields, $\mu_{\mathrm{e}(\mathrm{h})}<4 \times$ $10^{-7}\left(3 \times 10^{-8}\right) \mathrm{cm}^{2} / \mathrm{V} \cdot \mathrm{s}$, which would lead to ohmic losses and poor device performance.

The above assumptions on the nature of the diabatic states seem logical but are ultimately invalid. Indeed, if the diabatic states are constructed using the frontier orbitals of the entire BTDF molecule, the distributions of transfer integrals become significantly less broad and peak at much larger values, as shown in Figure $2 \mathrm{~b}$. As a result, predicted mobilities are much higher, $\mu_{\mathrm{e}(\mathrm{h})} \approx 5 \times 10^{-4}\left(10^{-5}\right) \mathrm{cm}^{2} / \mathrm{V} \cdot \mathrm{s}$, which is in agreement with experiments performed by admittance spectroscopy (AS).

2.2. Admittance Spectroscopy. AS allows to extract mobilities in an organic film sandwiched between two electrodes by applying $\mathrm{dc}$ and ac voltages and finding the transit time of the carriers in the film from a maximum in the negative differential susceptance $-\Delta B=\omega\left(C-C_{0}\right)$, where $C$ 
and $C_{0}$ are the $\omega$-dependent capacitance of the organic layer and its zero-frequency analogue ${ }^{37,38}$ (see the Supporting Information for details of device preparation and mobility extraction). Both experimental and simulated mobilities for electrons and holes are shown in Figure 3. The agreement is

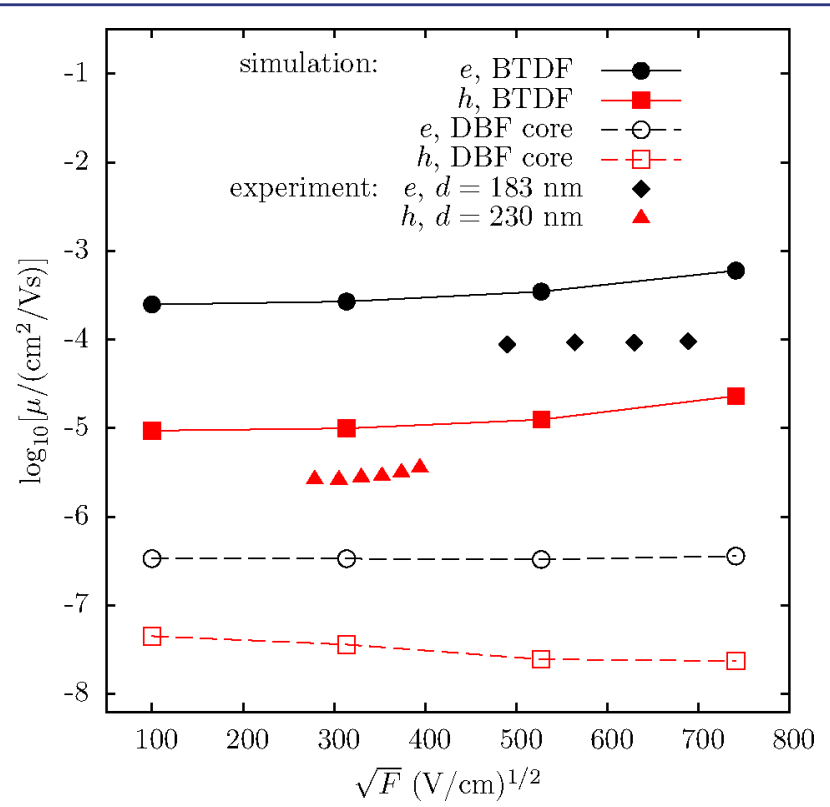

Figure 3. Simulated electron (black) and hole (red) mobility $\mu$ as function of applied field $F$, with diabatic states based on the whole BTDF molecule (solid) or the DBF core (dashed), respectively. For BTDF, error bars computed from a bootstrap analysis are smaller than the size of the data points. Experimental data obtained from admittance spectroscopy at room temperature on films of thickness $d$ are shown for comparison.

excellent for the relative electron/hole mobilities, and experimentally measured values are closer to the scenario where the substituents are incorporated in the diabatic states. Both simulations and experiments have small Poole-Frenkel slopes, indicating small energetic disorder. Simulated electron mobilities are higher due to stronger delocalization leading to $\lambda_{\mathrm{e}}$ $<\lambda_{\mathrm{h}}$, and $J_{\mathrm{e}}^{2} \gg J_{\mathrm{h}}{ }^{2}$, while $\sigma_{\mathrm{e}} \approx \sigma_{\mathrm{h}}$.

\section{DISCUSSION AND CONCLUSIONS}

We now discuss the discrepancy between the two approaches, that is, including/excluding the substituents in the definition of diabatic states. The reason for the much higher mobility in the first case is the mesomeric effect, which delocalizes the frontier orbitals over the silicon atom to the substituents, as shown in Figure 1d. A Mulliken population analysis ${ }^{39}$ indicates that a fraction of only 10 (4)\% of LUMO (HOMO) populates the substituents (see the Supporting Information). Although such small delocalization can be easily overlooked on a singlemolecule level, the effect on electronic couplings is much more pronounced since the substituents are in a closer contact than the cores. This is illustrated in the inset of Figure $2 b$, where the radial distribution function for centers of mass of phenyl rings is shown. Smaller separations boost electronic couplings exponentially and dramatically increase charge mobility.

The charge-transfer rate also depends on site energy differences. It is therefore essential to minimize variations of local dipole moments which lead to spatially correlated energetic disorder. ${ }^{40,41}$ Here the mesomeric effect is also beneficial, since it practically does not change the charge distribution of the conjugated core. One might argue that the mesomeric effect leads to an additional, conformational disorder of site and reorganization energies due to soft molecular degrees of freedom frozen in an amorphous morphology. This disorder is, however, small compared to the electrostatic disorder, is uncorrelated in space, and therefore has a minor effect on charge transport. ${ }^{17}$

To summarize, we suggest using the mesomeric effect to adjust the energy levels via side group attachments to conjugated cores. It substantially improves electronic couplings in the host by delocalizing the frontier orbitals, reduces reorganization energy, and does not lead to significant additional energetic disorder.

\section{ASSOCIATED CONTENT}

Supporting Information

Details of synthesis, MD simulations, force-field parameters, charge-transport simulations, and admittance spectroscopy. This material is available free of charge via the Internet at http://pubs.acs.org.

\section{AUTHOR INFORMATION}

\section{Corresponding Author}

christian.lennartz@basf.com; denis.andrienko@mpip-mainz. mpg.de

Notes

The authors declare no competing financial interest.

\section{ACKNOWLEDGMENTS}

This work was partially supported by the DFG program IRTG 1404, DFG grant SPP 1355, and BMBF grant MESOMERIE. We are grateful to Mara Jochum, Kostas Daoulas, Carl Poelking, Pascal Kordt, and Nicolle Langer for critical reading of the manuscript.

\section{REFERENCES}

(1) Forrest, S. R. Nature 2004, 428, 911-918.

(2) Reineke, S.; Lindner, F.; Schwartz, G.; Seidler, N.; Walzer, K.; Lussem, B.; Leo, K. Nature 2009, 459, 234-238.

(3) Giebink, N. C.; D’Andrade, B. W.; Weaver, M. S.; Brown, J. J.; Forrest, S. R. J. Appl. Phys. 2009, 105, 124514.

(4) Thompson, M. E.; Burrows, P. E.; Forrest, S. R. Curr. Opin. Solid State Mater. Sci. 1999, 4, 369-372.

(5) Erk, P.; Bold, M.; Egen, M.; Fuchs, E.; Gessner, T.; Kahle, K.; Lennartz, C.; Molt, O.; Nord, S.; Reichelt, H.; Schildknecht, C.; Johannes, H.-H.; Kowalsky, W. SID Symposium Digest of Technical Papers 2006, 37, 131-134.

(6) Holmes, R. J.; Forrest, S. R.; Tung, Y.-J.; Kwong, R. C.; Brown, J. J.; Garon, S.; Thompson, M. E. Appl. Phys. Lett. 2003, 82, 2422.

(7) Yersin, H. Top. Curr. Chem. 2004, 241, 1-26.

(8) Adachi, C.; Kwong, R. C.; Djurovich, P.; Adamovich, V.; Baldo, M. A.; Thompson, M. E.; Forrest, S. R. Appl. Phys. Lett. 2001, 79, 2082.

(9) Holmes, R. J.; D’Andrade, B. W.; Forrest, S. R.; Ren, X.; Li, J.; Thompson, M. E. Appl. Phys. Lett. 2003, 83, 3818.

(10) Sasabe, H.; Takamatsu, J.-i.; Motoyama, T.; Watanabe, S.; Wagenblast, G.; Langer, N.; Molt, O.; Fuchs, E.; Lennartz, C.; Kido, J. Adv. Mater. 2010, 22, 5003-5007.

(11) Hsieh, C.; Wu, F.; Fan, C.; Huang, M.; Lu, K.; Chou, P.; Yang, Y. O.; Wu, S.; Chen, I.; Chou, S.; Wong, K.; Cheng, C. Chem.-Eur. J. 2011, 17, 9180-9187.

(12) Turro, N. J.; Ramamurthy, V.; Scaiano, J. Modern Molecular Photochemistry of Organic Molecules; Palgrave Macmillan: 2010.

(13) Fuhrmann, T.; Salbeck, J. Adv. Photochem. 2002, 27, 83-166. 
(14) Nič, M., Jirát, J., Košata, B., Jenkins, A., McNaught, A., Eds.

IUPAC Compendium of Chemical Terminology, 2.1.0; IUPAC: Research Triagle Park, NC, ed., 2009.

(15) Marcus, R. A. Rev. Mod. Phys. 1993, 65, 599.

(16) Hutchison, G. R; Ratner, M. A.; Marks, T. J. J. Am. Chem. Soc.

2005, 127, 2339-2350.

(17) Rühle, V.; Lukyanov, A.; May, F.; Schrader, M.; Vehoff, T.; Kirkpatrick, J.; Baumeier, B.; Andrienko, D. J. Chem. Theory Comput.

2011, 7, 3335-3345.

(18) Rühle, V.; Junghans, C.; Lukyanov, A.; Kremer, K.; Andrienko,

D. J. Chem. Theory. Comput. 2009, 5, 3211-3223.

(19) Kirkpatrick, J.; Marcon, V.; Nelson, J.; Kremer, K.; Andrienko, D. Phys. Rev. Lett. 2007, 98, 227402.

(20) Feng, X.; Marcon, V.; Pisula, W.; Hansen, M. R.; Kirkpatrick, J.; Grozema, F.; Andrienko, D.; Kremer, K.; Müllen, K. Nat. Mater. 2009, $8,421-426$.

(21) Marcon, V.; Breiby, D. W.; Pisula, W.; Dahl, J.; Kirkpatrick, J.; Patwardhan, S.; Grozema, F.; Andrienko, D. J. Am. Chem. Soc. 2009, 131, 11426-11432.

(22) Olivier, Y.; Muccioli, L.; Lemaur, V.; Geerts, Y. H.; Zannoni, C.; Cornil, J. J. Phys. Chem. B 2009, 113, 14102-14111.

(23) Di Donato, E.; Fornari, R. P.; Di Motta, S.; Li, Y.; Wang, Z.; Negri, F. J. Phys. Chem. B 2010, 114, 5327-5334.

(24) May, F.; Marcon, V.; Hansen, M. R.; Grozema, F.; Andrienko, D. J. Mater. Chem. 2011, 21, 9538.

(25) Kwiatkowski, J. J.; Nelson, J.; Li, H.; Brédas, J. L.; Wenzel, W.; Lennartz, C. Phys. Chem. Chem. Phys. 2008, 10, 1852.

(26) Nelson, J.; Kwiatkowski, J. J.; Kirkpatrick, J.; Frost, J. M. Acc. Chem. Res. 2009, 42, 1768-1778.

(27) Nagata, Y.; Lennartz, C. J. Chem. Phys. 2008, 129, 034709.

(28) Lukyanov, A.; Andrienko, D. Phys. Rev. B 2010, 82, 193202.

(29) Vehoff, T.; Baumeier, B.; Troisi, A.; Andrienko, D. J. Am. Chem. Soc. 2010, 132, 11702-11708.

(30) Rühle, V.; Kirkpatrick, J.; Andrienko, D. J. Chem. Phys. 2010, $132,134103$.

(31) Vukmirović, N.; Wang, L.-W. Nano Lett. 2009, 9, 3996-4000.

(32) Brédas, J.-L.; Beljonne, D.; Coropceanu, V.; Cornil, J. Chem. Rev.

2004, 104, 4971-5004.

(33) Thole, B. Chem. Phys. 1981, 59, 341-350.

(34) Ponder, J. W.; Wu, C.; Ren, P.; Pande, V. S.; Chodera, J. D.; Schnieders, M. J.; Haque, I.; Mobley, D. L.; Lambrecht, D. S.; DiStasio, R. A.; Head-Gordon, M.; Clark, G. N. I.; Johnson, M. E.; HeadGordon, T. J. Phys. Chem. B 2010, 114, 2549-2564.

(35) Valeev, E. F.; Coropceanu, V.; da Silva Filho, D. A.; Salman, S.; Brédas, J.-L. J. Am. Chem. Soc. 2006, 128, 9882-9886.

(36) Baumeier, B.; Kirkpatrick, J.; Andrienko, D. Phys. Chem. Chem. Phys. 2010, 12, 11103.

(37) Blom, P.; Martens, H.; Huiberts, J. Synth. Met. 2001, 121, 16211624.

(38) Tsang, S. W.; So, S. K.; Xu, J. B. J. Appl. Phys. 2006, 99, 013706-013706-7.

(39) Mulliken, R. S. J. Chem. Phys. 1955, 23, 1833.

(40) Novikov, S. V.; Vannikov, A. V. J. Phys. Chem. 1995, 99, 1457314576 .

(41) Novikov, S. V.; Dunlap, D. H.; Kenkre, V. M.; Parris, P. E.; Vannikov, A. V. Phys. Rev. Lett. 1998, 81, 4472. 